\title{
THE SEMANTIC ARCHITECTURE OF THE DOMAIN OF EXISTENCE IN THE FRENCH AND ENGLISH LANGUAGES
}

\author{
Isabel Negro \\ Universidad Complutense
}

\begin{abstract}
ABSRACT: In this paper we will analyse the semantic domain of EXISTENCE in the French and English languages following the Functional-Lexematic Model (Martin Mingorance 1984), which consists of four levels of lexical description: paradigmatic, syntagmatic, pragmatic, and cognitive. We will focus on the paradigmatic, syntagmatic and cognitive axes.

On the paradigmatic axis the lexemes are grouped under semantic domains and dimensions elaborated on the basis of shared meaning components following Dik's method of Stepwise Lexical Decomposition (1978b). The relevant semantic parameters are those of manner, means/instrument, place, time, and the parameter describing the nature of the subject/object.

On the syntagmatic axis we specify the syntactic patterns of each lexeme following Dik's model (1978a). Three complementation patterns govern this semantic domain: SV, SVAdjunct, and SVO (NP).

On the cognitive axis we examine the connections of EXISTENCE with other domains: PERCEPTION, FEELING, ACTION, COGNITION, CHANGE, POSITION and MOVEMENT.
\end{abstract}

RESUMEN: En este artículo analizaremos el dominio semántico de la EXISTENCIA en francés e inglés, siguiendo el Modelo Lexemático-Funcional (Martín Mingorance 1984), que consta de cuatro ejes de descripción : paradigmático, sintagmático, pragmático y cognitivo. Nos centraremos en los ejes paradigmático, sintagmático y cognitivo

En el eje paradigmático los lexemas están agrupados en dominios y dimensiones en función de rasgos de significado comunes. El diseño de las dimensiones atiende al mecanismo de la Descomposición Léxica Gradual de Dik (1978b) . Los parámetros semánticos opositivos son los de modo, medio/instrumento, lugar, tiempo, y aquel que describe la naturaleza del sujeto/objeto.

En el eje sintagmático especificamos la sintaxis léxica de los verbos según el modelo de Dik (1978a). Tres patrones sintácticos rigen este dominio: SV, SVCC y SVO (SN).

En el eje cognitivo estudiamos las relaciones semánticas del dominio de la EXISTENCIA con otros dominios: PERCEPCION, SENTIMIENTO, ACCION, COGNICION, CAMBIO, POSICION Y MOVIMIENTO.

\section{Introduction}

EXISTENCE is one of the most basic lexical domains. In this paper we shall contrast the semantic configuration of this field in the French and English languages 
following the Functional-Lexematic model elaborated by Martín Mingorance (1984; 1985a,b; 1987a,b,c; 1990). We will expound the similarities and divergences between the French and English domains of EXISTENCE on three levels: paradigmatic, syntagmatic and cognitive.

\section{An outline of the Functional-Lexematic model}

The Functional-Lexematic model integrates Coseriu's Lexematics (1977) and Dik's Functional Grammar (1978a), and consists of four levels of lexical analysis: paradigmatic, syntagmatic, pragmatic and cognitive.

The paradigmatic axis is concerned with the semantic description of the lexemes according to the principles of opposition and functionality. The lexemes are grouped under semantic domains ${ }^{1}$, which are in turn divided into dimensions 2 following the postulates of Coseriu's Lexematics. Each dimension subsumes a set of semantically closed lexemes and represents an area of meaning within the general concept embodied by the domain.

The method used in the elaboration of the meaning definitions is Dik's Stepwise Lexical Decomposition (1978b), according to which each lexeme is made up of an information nucleus, the definiens, and a set of relevant features which mark the distance from the other members of the dimension and the field. The lexical unit which constitutes the act nucleus of the dimension is the archilexeme. Then, Faber and Mairal (1994: 13-14) claim that "lexical dimensions in each field are established in terms of oppositions formulated from the definitional structure of the lexical units. These oppositions characterize both the internal structure of the dimension in question as well as the lexical structure of the items that it contains. Lexical dimensions are thus directly derived from the definitional structure of lexical units".

On the syntagmatic axis we specify the syntactic patterns of the predicates in the dimension adopting Dik's predicate frames model as a notational device (1978b). The predicate frames are formal structures including these types of information:

(i) The form of the predicate

(ii) The syntactic category to which it belongs

1. The following semantic fields can be distinguished (Faber and Mairal 1992): EXISTENCE, MOVEMENT, POSITION, CHANGE, PERCEPTION, FEELING, COGNITION, POSSESSION, SPEECH, SOUND, and GENERAL ACTION.

2. This structural level is described by Geckeler (1977). A dimension can be thought of as a viewpoint of lexical articulation which operates in a lexical field and activates oppositions between certain lexemes within that field. 
(iii) Its quantitative valency 3 , i.e. the number of arguments that the predicate requires.

(iv) Its qualitative valency, i.e. the semantic functions of the arguments and the selection restrictions holding for them.

(v) Meaning definition

Predicate frames describe a state of affairs and specify the relationship between the predicate arguments (represented by the variable $x$ ). Each argument is characterized by a selection restriction - described in terms of binary semantic features - and fulfils a semantic function (Agent, Force, Experiencer, Goal, Recipient, etc.).

Consider the predicate frame of the verb prononcer:

$\left[\left(\mathrm{x}_{1} \text { : prototyp. human }\right)_{\mathrm{Ag}}\left(\mathrm{x}_{2} \text { : prototyp. -concrete } \varepsilon \text { sounds, letters, words }\right)_{\mathrm{Go}}\right]_{\text {Action }}$

This frame describes an Action (a state of affairs defined by the parameters [+control, +dynamism] and specifies the relationship between a human argument, performing the function of Agent, and an argument fulfilling the function of Goal and semantically marked as [-concrete].

The pragmatic axis deals with the meaning components that provide information about the communicative situation and about the way in which speakers perceive and evaluate the world. In this light, it is safe to affirm that most lexical models focus on descriptive meaning, neglecting subjective and connotative factors. However, in Lyons' terms (1977), words also carry social and affective meaning.

The description of the cognitive axis is based on the idea that semantic structure reflects conceptual structure ${ }^{4}$. Then starting from the postulate that each semantic domain represents a basic conceptual category, we can arrive at the delineation of conceptual schemata. Faber and Mairal (1998:19) define a schema in the following terms:

"A schema is a modular, dynamic characterization that subsumes linguistic symbolic units obtained in a bottom-to-top fashion through the activation of lowerlevel schemata. These schemata are linguistically motivated and reflect our understanding of reality".

Modular means that a given schema includes a number of opposing subschemata. Dynamic suggests that cognitive schemata are linked to other schemata. Linguistic entails that the units which define a cognitive schema obtain from semantic structure.

3. Following Sommers (1984: 508), "valency is concerned with relationships between the verbal predicate and the other elements making up a predication. These elements divide up into those which are closely associated with the predicate, termed "complements", and the rest, termed "adjuncts". This distinction correlates with Dik's distinction between arguments and satellites.

4. The argument that semantic structure reflects conceptual structure is found in Faber (1994a), Faber \& Mairal (1994,1998), Faber \& Pérez (1993), and Lakoff (1987). 
The connections between cognitive schemata embody metaphoric and metonymic processes and are expressed in a semantic macronet (cf. below).

\section{Contrastive paradigmatic analysis}

The lexemes which integrate the French and English fields of EXISTENCE can be classified according to semantic parameters (cf. appendix 1). The semantic features which are relevant to the paradigmatic structure of both lexical domains are those of manner, means/instrument, time, place, and that describing the nature of the object.

The parameters of manner and means/instrument are relevant to the definitional structure of both the French and English semantic fields.

The parameter of manner is particularly relevant in the establishment of the semantic hierarchies of the verbs under the causative subdimensions Faire mourir qqn/un animal / To cause sb to die and Faire disparaître complètement qqch / To cause sth to disappear. The verbs assommer, exécuter, lyncher, étrangler, étouffer, empoisonner, lapider, fusiller and décapiter all denote different ways of killing someone/an animal, sharing with the English terms an element of manner.

In the subdimension Faire mourir qqn/un animal instrument is a meaningful feature of the lexemes égorger, poignarder and guillotiner.

On the other hand, both French and English definitions in the dimensions Exister en tant que représentation de qqch / To exist as a representation of sth are arranged in terms of means, although this parameter is more salient in English because the English dimension subsumes more verbs.

The relevance of the time and place parameters results from the way of conceptualizing existence. Something can exist in the objective world over a particular space and/or a particular period of time.

Further, the parameter of time is a structuring device within both semantic domains. Some dimensions are organized in terms of time: Exister dans le temps / To exist in time, Continuer à exister dans le temps / To continue to exist in time, and Cesser d'exister dans le temps / To stop happening.

The time parameter is most central to the definition structure of the French verbs in the dimension Continuer à exister dans le temps, whereas in the correlative English dimension this semantic pattern is made explicit in only two verbs (linger, persist).

Another correspondence between the French and English field of EXISTENCE can be pinpointed in relation with the parameter describing the nature of the subject. Below we present the lexemes of both domains where this parameter is relevant within their definition:

(i) Animate entity:

- Human beings: se reproduirel/reproduce, procréer/procreate.

- Animals: éclore /hatch, croître. 
- Plants: fleurir, croître, pousser.

(ii) Inanimate entity:

- Ideas / feelings: s'éveiller, percer, germer.

- Daylight: poindre, pointer, dawn.

- Sun / moon / stars: se lever, paraître ${ }^{2}$, pointer, percer.

- Noise / voice: s'élever.

- Activity: fleurir, prospérer.

- Rash/sore: erupt ${ }^{2}$

Note that the parameter describing the nature of the subject is more basic in French.

The major divergence between the French and English lexical fields concerns the semantic differentiation pattern nature/quality of the object. The parameter describing the nature of the object is salient in English, whereas that describing the quality of the object is more relevant in French.

\section{Contrastive syntagmatic analysis}

An examination of the syntactic properties of the French and English predicates of EXISTENCE reveals that they have a limited number of complementation patterns. We have pinned down the following predicate schemata (cf. above)

\subsection{SV5}

The predicate frames for this syntactic pattern have the following formats:

1. $\left[\left(\mathrm{x}_{1} \text { : prototyp. living/human }\right)_{\text {Proc }}\right]_{\text {Process }}$

This frame designates a Process qualified by a Processed argument prototypically living/human.

\begin{tabular}{|l|l|}
\hline French predicates & English predicates \\
\hline $\begin{array}{l}\text { fleurir, s'épanouir, grandir, croître, pousser } \\
\text { ressusciter }{ }^{2} \text {, mourir, décéder, claquer, crever } \\
\text { casser sa pipe, passer l'arme à gauche, manger } \\
\text { les pissenlits par la racine, avaler son bulletin de } \\
\text { naissance / sa chique, sortir les pieds devant } \\
\text { sortir entre quatre planches, boire le bouillon } \\
\text { d'onze heures, déposer le bilan, faire couic } \\
\text { ramasser ses outils, faire sa malle / sa valise } \\
\text { aller chez les taupes, périr, expirer', se noyer } \\
\text { s'électrocuter }\end{array}$ & \\
\hline
\end{tabular}

5. The linguistic symbols used for the syntactic functions of the clausal elements are $S=$ Subject, V=Verb, $\mathrm{O}=$ Object. The label "Adjunct" (Quirk 1985) refers to the grammatical function of adverbial. Adjuncts can fulfil various semantic roles (place, time, manner, etc.). On the other hand, each syntactic function can have different realizations. For example, an object can be realized as a noun phrase (NP) or as a nominal clause. 


\section{2. $\left[\left(\mathrm{x}_{1} \text { : prototyp. living }\right)_{\mathrm{Ag}}\right]_{\text {Action }}$}

This schema describes an Action encoding a living argument fulfilling the semantic function of Agent.

- French predicates: éclore, avorter, faire une fausse couche, se suicider, se brûler la cervelle, disparaître, s'evaporer, se volatiliser, s'évanouir.

- English predicates: reproduce1, procreate, hatch, miscarry, commit suicide.

3. $\left[\left(\mathrm{x}_{1} \text { : prototyp. -concrete }\right)_{\mathrm{Fo}}\right]_{\text {Process }}$

This predication describes a Process qualified by a Force argument which has the following selection restrictions:

\begin{tabular}{|c|c|c|}
\hline Selection restriction & French predicates & English predicates \\
\hline Theories/Institutions & & be born ${ }^{1}$, originate ${ }^{1}$, flourish \\
\hline Feeling/emotion/attitude & disparaître, s'évanouir & $\begin{array}{l}\text { germinate }{ }^{1} \text {, dawn, surface } \\
\text { materialize, pass away/on } \\
\text { disappear, evaporate, vanish } \\
\text { fade, dissipate }\end{array}$ \\
\hline $\begin{array}{l}\text { Fact }<\text { situation, process, } \\
\text { event, action }>\end{array}$ & $\begin{array}{l}\text { commencer }{ }^{13} \text {, se produire } \\
\text { arriver, se passer, survenir } \\
\text { finir, cesser, (se) terminer } \\
\text { s'achever }\end{array}$ & $\begin{array}{l}\text { start }^{13}, \text { resume, ensue } \\
\text { originate }^{1} \text {, threaten, open } \\
\text { come up, crop up }\end{array}$ \\
\hline Intellect & se matérialiser, s'évanouir & $\begin{array}{l}\text { germinate }^{1}, \text { miscarry } \\
\text { pass away/on }\end{array}$ \\
\hline Sound & & fade \\
\hline Business/activity & se développer, prospérer & \\
\hline Term of office/contract & expirer $^{2}$ & expire $^{2}$, lapse \\
\hline
\end{tabular}

\subsection{SVAdjunct}

The predicate frames for this government pattern have the following readings:

1. $\left[\left(\mathrm{x}_{1} \text { : prototyp. human/living }\right)_{\mathrm{Proc}}\right.$

$\left.\left(\mathrm{y}_{1} \text { : prototyp. time, place, manner }\right)_{\mathrm{Ti} / \mathrm{Loc} / \mathrm{Ma}}\right]_{\text {Process }}$

This schema designates a Process and brings to light a human/living Processed and a satellite which can perform various functions. The selection restrictions touching upon the arguments are sketched below. 


\begin{tabular}{|c|c|c|c|}
\hline & Selection restriction & French predicates & English predicates \\
\hline \multirow[t]{2}{*}{ Subject } & Human & $\begin{array}{l}\text { naître }{ }^{12} \text {, éclore } \\
\text { vivoter, végéter } \\
\text { grandir, décéder } \\
\text { expirer }{ }^{1}, \text { se noyer } \\
\text { se tuer, } \mathrm{s}^{\prime} \text { électrocuter }\end{array}$ & $\begin{array}{l}\text { die, expire }{ }^{1} \text {, perish } \\
\text { suffocate }^{1} \text {, fade }\end{array}$ \\
\hline & Living & $\begin{array}{l}\text { se reproduire }{ }^{1} \\
\text { se multiplier } \\
\text { se propager, éclore } \\
\text { naître }^{1}, \text { vivre, croître } \\
\text { pousser, fleurir } \\
\text { mourir, périr }\end{array}$ & grow, starve ${ }^{1}$ \\
\hline \multirow{3}{*}{ Adjunct } & Time & $\begin{array}{l}\text { naître }{ }^{1} \text {, vivre, fleurir } \\
\text { éclore, mourir, périr } \\
\text { décéder, expirer }^{1}\end{array}$ & $\begin{array}{l}\text { die, expire }{ }^{1} \text {, perish } \\
\text { suffocate }^{1}, \text { starve }^{1}\end{array}$ \\
\hline & Place & $\begin{array}{l}\text { naître }{ }^{1} \text {, vivre, croître } \\
\text { pousser, s'épanouir } \\
\text { mourir, se noyer }\end{array}$ & $\begin{array}{l}\text { grow, expire }{ }^{1} \text {, perish } \\
\text { suffocate }^{1} \text {, die, } \text { starve }^{1} \\
\text { fade }\end{array}$ \\
\hline & Manner & $\begin{array}{l}\text { se reproduire } \\
\text { se multiplier } \\
\text { se propager, naître }{ }^{2} \\
\text { vivre, végéter, vivoter } \\
\text { grandir, mourir, périr } \\
\text { se tuer, s'électrocuter }\end{array}$ & perish \\
\hline
\end{tabular}

2. $\left[\left(\mathrm{x}_{1} \text { : prototyp.+concrete }\right)_{\mathrm{Fo}}\left(\mathrm{y}_{1} \text { : prototyp. time, place, cause, manner }\right)_{\mathrm{Ti} / \mathrm{Loc} /}\right.$ $\mathrm{Cau} / \mathrm{Ma}]_{\text {Process }}$

This frame differs from the one above in the semantic role of the first argument (Force), semantically marked as concrete.

Below we specify the semantic feature of the arguments:

a) Force:

- "Entity / object": evaporate, appear, disappear.

- "Something visible": appear / apparaître, se former, surgir, materialize, disappear, vanish.

- "Liquid / gas": jaillir

- "Volcano": erupt1

- "Machines": die.

- "Documents": expire2 / expirer2.

- "Speech / writing": end.

- "Light": poindre, pointer.

- "Sun / moon / stars": se lever, paraître ${ }^{2}$, pointer, percer.

- "Day": poindre. 
b) Satellite:

- Place: materialize, erupt1, appear/ apparaître, se former, surgir, jaillir, poindre, pointer, se lever, paraître2, die.

- Time: se former, erupt1, expire2 / expirer2.

- Cause: disappear, vanish.

- Manner: end

3. $\left[\left(\mathrm{x}_{1} \text { : prototyp. -concrete }\right)_{\mathrm{Fo}}\left(\mathrm{y}_{1} \text { : prototyp. time, place, manner }\right)_{\mathrm{Ti} / \mathrm{Loc} / \mathrm{Ma}}\right]_{\text {Process }}$

This frame resembles the previous one in that it encodes a Force argument and a satellite, but differs from it in the semantic scope of the subject argument, characterized as [-concrete] ("theory", "fact", "feeling", "sound", "activity"). The satellite may fulfil three semantic roles (time, place, manner).

\begin{tabular}{|c|c|c|c|}
\hline Syntactic function & Selection restriction & French predicates & English predicates \\
\hline \multirow{5}{*}{ Subject } & $\begin{array}{l}\text { Theories/ideas/ } \\
\text { institutions }\end{array}$ & s'éveiller, germer & originate $^{\mathrm{l}}$, perish \\
\hline & $\begin{array}{l}\text { Fact < situation, action } \\
\text { event, process > }\end{array}$ & $\begin{array}{l}\text { se produire, arriver } \\
\text { se passer, survenir } \\
\text { avoir lieu, éclater } \\
\text { se reproduire } 2 \text {, finir } \\
\text { cesser, (se) terminer } \\
\text { s'achever }\end{array}$ & $\begin{array}{l}\text { happen, take place } \\
\text { transpire, occur, rage } \\
\text { end, conclude } \\
\text { terminate }\end{array}$ \\
\hline & $\begin{array}{l}\text { Feeling/emotion/ } \\
\text { behaviour }\end{array}$ & $\begin{array}{l}\text { germer, éclater } \\
\text { exploser, se déchaîner }\end{array}$ & erupt $^{1}$, die, perish \\
\hline & Sound & s'élever & \\
\hline & Activity & prospérer & \\
\hline \multirow{3}{*}{ Adjunct } & Time & $\begin{array}{l}\text { éclater, exploser } \\
\text { se déchaîner } \\
\text { s'éveiller, arriver } \\
\text { se produire, se passer } \\
\text { survenir, avoir lieu } \\
\text { se reproduire', cesser } \\
\text { prospérer, s'achever } \\
\text { (se) terminer, finir }\end{array}$ & $\begin{array}{l}\text { happen, take place } \\
\text { transpire, conclude } \\
\text { terminate, perish }\end{array}$ \\
\hline & Place & $\begin{array}{l}\text { éclater, s'élever } \\
\text { germer, s'éveiller } \\
\text { se produire, se passer } \\
\text { survenir, avoir lieu }\end{array}$ & $\begin{array}{l}\text { happen, take place } \\
\text { transpire, erupt }{ }^{1} \text {, die } \\
\text { perish }\end{array}$ \\
\hline & Manner & & $\begin{array}{l}\text { happen, take place } \\
\text { transpire, end } \\
\text { conclude, die, perish }\end{array}$ \\
\hline
\end{tabular}


4. $\left[\left(\mathrm{x}_{1} \text { : prototyp. -concrete) }\left(\mathrm{y}_{1} \text { : prototyp. time, place }\right)_{\mathrm{Ti} / \text { Loc }}\right]_{\text {State }}\right.$

This predication designates a State and codifies a subject argument which has no semantic function, and a satellite which fulfils the semantic role of time or place. The selection restrictions holding upon the two arguments are described below.

\begin{tabular}{|l|l|l|l|}
\hline Syntactic function & Selection restriction & French predicates & English predicates \\
\hline \multirow{5}{*}{ Subject } & $\begin{array}{l}\text { Theories / institutions } \\
\text { ideas }\end{array}$ & $\begin{array}{l}\text { prevail, live, live on } \\
\text { survive, endure } \\
\text { remain, last, linger } \\
\text { persist, go on }\end{array}$ \\
\cline { 2 - 4 } & $\begin{array}{l}\text { Fact < event, situation } \\
\text { process, action }>\end{array}$ & $\begin{array}{l}\text { commencer } \\
\text { se reproduire } \\
\text { continuer, durer } \\
\text { se prolonger, traîner }\end{array}$ & go on, rage \\
\cline { 2 - 5 } & Feeling/ emotion & $\begin{array}{l}\text { live, endure, remain } \\
\text { last, linger, persist }\end{array}$ \\
\cline { 2 - 5 } & Customs & subsister, se perpétuer & prevail \\
\cline { 2 - 5 } & Time & s'écouler, passer & $\begin{array}{l}\text { prevail, live, live on } \\
\text { survive, remain, last } \\
\text { linger, go on, rage }\end{array}$ \\
\hline \multirow{3}{*}{ Adjunct } & Time & $\begin{array}{l}\text { commencer }{ }^{13} \\
\text { continuer, durer } \\
\text { traîner }\end{array}$ & $\begin{array}{l}\text { prevail, live, live on } \\
\text { survive, go on, rage }\end{array}$ \\
\hline
\end{tabular}

\subsection{SVO (NP)}

The predicate frames for this complementation pattern go as follows:

1. $\left[\left(\mathrm{x}_{1} \text { : prototyp. human }\right)_{\mathrm{Ag}}\left(\mathrm{x}_{2} \text { : prototyp. human/living }\right)_{\mathrm{Go}}\right]_{\mathrm{Action}}$

This schema describes an Action and specifies the relation between an Agent, prototypically human, and a Goal.

The semantic scope of the object is taken up below. 


\begin{tabular}{|c|c|c|}
\hline Sel. restrict. on the object & French predicates & English predicates \\
\hline Human & $\begin{array}{l}\text { procréer, engendrer, accoucher } \\
\text { donner le jour, enfanter } \\
\text { avoir/ faire la peau de } \\
\text { assassiner, zigouiller, lapider } \\
\text { massacrer, exterminer, liquider } \\
\text { exécuter, fusiller, décapiter } \\
\text { guillotiner, pendre, crucifier } \\
\text { lyncher, étrangler, étouffer } \\
\text { empoisonner, poignarder }\end{array}$ & $\begin{array}{l}\text { beget }^{2} \text {, sire, give birth to } \\
\text { deliver, remove, do away with } \\
\text { eliminate, do in, bump off } \\
\text { liquidate, crucify, lynch } \\
\text { execute, hang, murder } \\
\text { assassinate, starve }{ }^{2} \text {, smother } \\
\text { gas, drown }{ }^{2} \text {, slay, strangle } \\
\text { suffocate }^{2}\end{array}$ \\
\hline Living & $\begin{array}{l}\text { propager, tuer, abattre } \\
\text { assommer, égorger, se pendre }\end{array}$ & $\begin{array}{l}\text { kill, massacre, slaughter } \\
\text { exterminate, butcher, destroy }{ }^{1}\end{array}$ \\
\hline
\end{tabular}

2. $\left[\left(\mathrm{x}_{1} \text { : prototyp. human/living }\right)_{\mathrm{Ag}}\left(\mathrm{x}_{2} \text { : prototyp. +concrete }\right)_{\mathrm{Go}}\right]_{\text {Action }}$

This predication differs from the one above in the semantic path of the Goal, semantically characterized as concrete ("object", "writing/picture", "money", "food / manufactured goods", "place").

\begin{tabular}{|c|c|c|}
\hline Sel. restrict. on the object & French predicates & English predicates \\
\hline Object & $\begin{array}{l}\text { produire }{ }^{1} \text {, pondre, reproduire } \\
\text { contrefaire, enlever, gratter }\end{array}$ & $\begin{array}{l}\text { make }^{2} \text {, create, produce }{ }^{1} \text { lay } \\
\text { spawn, duplicate, reproduce } \\
\text { fake, forge, counterfeit, bear } \\
\text { kill, destroy }{ }^{2}\end{array}$ \\
\hline Writing/picture & $\begin{array}{l}\text { falsifier, copier, contrefaire } \\
\text { imiter, attaquer, effacer } \\
\text { raturer, rayer, barrer }\end{array}$ & $\begin{array}{l}\text { copy, duplicate, reproduce }{ }^{2} \\
\text { forge, counterfeit, delete, erase } \\
\text { cancel, efface }\end{array}$ \\
\hline Money & falsifier, contrefaire, imiter & forge, counterfeit \\
\hline Food / manufactured goods & produire $^{1}$ & produce $^{1}$ \\
\hline Place & détruire & destroy $^{2}$ \\
\hline
\end{tabular}

3. $\left[\left(\mathrm{x}_{1} \text { : prototyp. human }\right)_{\mathrm{Ag}}(\mathrm{x} 2 \text { : prototyp. -concrete })_{\mathrm{Go}}\right]_{\mathrm{Action}}$

Here the Goal argument is semantically marked as [-concrete]. This semantic feature is further specified in the table below. 


\begin{tabular}{|c|c|c|}
\hline Sel. restrict. on the object & French predicates & English predicates \\
\hline $\begin{array}{l}\text { Fact }<\text { situation, process, } \\
\text { event, action }>\end{array}$ & $\begin{array}{l}\text { créer, commencer }{ }^{2} \text {, amorcer } \\
\text { engager, entamer, entreprendre } \\
\text { inaugurer, ressusciter }{ }^{1} \\
\text { recommencer }\end{array}$ & $\begin{array}{l}\text { make }^{2} \text {, reproduce } \\
\text { resume, bring about } \\
\text { precipitate, induce, provoke } \\
\text { prompt, effect, occasion, start }{ }^{2} \\
\text { commence }^{2} \text {, institute, instigate } \\
\text { loose, open }^{2} \text {, originate } \\
\text { inaugurate, initiate, kill } \\
\text { eradicate, remove, efface } \\
\text { expunge }\end{array}$ \\
\hline Feeling/sensation/attitude & & $\begin{array}{l}\text { create, make }{ }^{2} \text {, engender } \\
\text { ressurrect, fake, produce } \\
\text { germinate }^{2}, \text { precipitate, induce } \\
\text { provoke, prompt, kill, erase } \\
\text { eradicate, destroy, obliterate } \\
\text { remove, efface, expunge }\end{array}$ \\
\hline Intellect & & $\begin{array}{l}\text { produce }^{2}, \text { germinate } \\
\text { obliterate, erase } \\
\text { obiginate } e^{2} \text {, efface }\end{array}$ \\
\hline $\begin{array}{l}\text { System/organization/ } \\
\text { institution/law }\end{array}$ & $\begin{array}{l}\text { créer, constituer, former } \\
\text { fonder, instaurer, instituer }\end{array}$ & $\begin{array}{l}\text { establish, found, institute } \\
\text { open }^{2}, \text { destroy }^{2}\end{array}$ \\
\hline Behaviour & & copy, reproduce ${ }^{2}$ \\
\hline Information & & erase \\
\hline Result/state & & create, engender, produce ${ }^{2}$ \\
\hline Business/company & liquider & start $^{2}{ }^{4}$, liquidate \\
\hline
\end{tabular}

4. $\left[\left(\mathrm{x}_{1} \text { : prototyp. human }\right)\left(\mathrm{x}_{2} \text { : prototyp. -concrete }\right)\right]_{\text {State }}$

This is a two-place predication describing a State. The semantic scope of the complement is described below:

- "Feature / value / quality": represent, stand for, exemplify, personify1, personnifier, typify, embody / incarner.

- "Meaning / representation": represent / représenter, mean, signify, personify ${ }^{1}$

- "Feeling / attitude": typify, depict.

- "Intellect": represent / représenter, stand for, exemplify, instance, depict, embody.

- "Fact": exemplify, depict. 
5. $\left[\left(\mathrm{x}_{1} \text { : prototyp. +concrete }\right)\left(\mathrm{x}_{2} \text { : prototyp. -concrete }\right)\right]_{\text {State }}$

This schema differs from the one above in the selection restriction holding for the subject argument.

The semantic delineation of the arguments goes as follows:

a) Subject

- “Object": represent / représenter, mean, exprimer, symboliser.

- "Symbol / sign / gesture / facial expression": represent, mean, signify, indicate, indiquer, symbolize, denote / dénoter

- "Word / name": mean.

b) Object:

- "Meaning / representation": represent / représenter, mean, signify, indicate, indiquer, symbolize, denote / dénoter.

- "Feature / value / quality": represent, mean, indicate, symbolize, denote / dénoter, indiquer.

- "Intellect": represent, exprimer, symboliser.

- "Fact": mean.

6. $\left[\left(\mathrm{x}_{1} \text { : prototyp. -concrete) }\left(\mathrm{x}_{2} \text { : prototyp. -concrete }\right)\right]_{\text {State }}\right.$

This frame also designates a State whose arguments are semantically marked as [-concrete]:

a) Subject:

- "Fact": mean, indicate / indiquer, symbolize, denote, typify.

- "Intellect": represent.

- "Feeling / attitude": mean, indicate / indiquer, symbolize, denote, typify.

- "Organization": stand for.

b) Object:

- "Meaning": mean, indicate, symbolize, denote.

- "Value / quality": stand for, indicate, symbolize, denote, typify.

- "Intellect": represent, stand for.

- "Feeling / attitude": mean.

7. $\left[\left(\mathrm{x}_{1} \text { : prototyp. -concrete }\right)_{\mathrm{Fo}}\left(\mathrm{x}_{2}: \text { prototyp. -concrete }\right)_{\mathrm{Go}}\right]_{\text {Process }}$

This frame designates a Process and specifies the relation between a Force argument and a Goal argument, both semantically characterized as [-concrete].

Let us present the selection restrictions imposed upon the two arguments. 


\begin{tabular}{|c|c|c|c|}
\hline Syntactic function & Selection restriction & French predicates & English predicates \\
\hline \multirow[t]{3}{*}{ Subject } & $\begin{array}{l}\text { Fact <situation,action } \\
\text { process, event }>\end{array}$ & $\begin{array}{l}\text { produire }{ }^{2} \text {, provoquer } \\
\text { susciter, causer } \\
\text { motiver, engendrer }{ }^{2} \\
\text { occasionner } \\
\text { déchainer, entraîner }\end{array}$ & $\begin{array}{l}\text { create, spawn, } \\
\text { produce }{ }^{2}, \text { bring about } \\
\text { precipitate, spark, } \\
\text { induce, provoke, } \\
\text { prompt, occasion } \\
\text { give birth to }\end{array}$ \\
\hline & Feeling/attitude & $\begin{array}{l}\text { susciter, motiver } \\
\text { engendrer }{ }^{2} \text {, déchaîner }\end{array}$ & \\
\hline & Theories/institutions & & $\begin{array}{l}\text { institute, loose, } \\
\text { initiate, do away with }\end{array}$ \\
\hline \multirow[t]{5}{*}{ Object } & $\begin{array}{l}\text { Fact < situation, action } \\
\text { process, event }>\end{array}$ & $\begin{array}{l}\text { produire }{ }^{2} \text {, causer } \\
\text { provoquer, susciter } \\
\text { motiver, occasionner } \\
\text { entraîner }\end{array}$ & $\begin{array}{l}\text { bring about } \\
\text { precipitate, spark } \\
\text { induce, provoke } \\
\text { prompt, occasion } \\
\text { give birth to, institute } \\
\text { initiate, do away with }\end{array}$ \\
\hline & Feeling / attitude & $\begin{array}{l}\text { susciter, engendrer }{ }^{2} \\
\text { déchaîner }\end{array}$ & $\begin{array}{l}\text { create, produce }{ }^{2}, \\
\text { precipitate, spark } \\
\text { induce, provoke } \\
\text { prompt }\end{array}$ \\
\hline & Institution/organiz. & & haunt \\
\hline & Result / state & & $\begin{array}{l}\text { create, produce }{ }^{2} \\
\text { spawn }\end{array}$ \\
\hline & Expenses & occasionner & \\
\hline
\end{tabular}

\subsection{SVO (Passive)}

The predicate frame for this syntactic pattern has the following reading:

$\left[\left(\mathrm{x}_{1} \text { : prototyp. human }\right)_{\mathrm{Ag}}\left(\mathrm{x}_{2} \text { : prototyp. human/animal }\right)_{\mathrm{Go}}\right]_{\mathrm{Action}}$

This schema describes an Action qualified by a human Agent and a Goal argument, prototypically living.

It is the predication of the verbs tuer, abattre, assommer, égorger, assassiner, zigouiller, massacrer, exterminer, liquider, exécuter, fusiller, behead, decapitate, décapiter, guillotine, guillotiner, pendre, crucifier, electrocute, lyncher, étrangler, étouffer, empoisonner, lapider, poignarder.

\subsection{SVO (Nominal clause)}

The predicate frames for this government pattern have the following forms:

1. $\left[\left(\operatorname{Cert~}_{1}:\left[\operatorname{Pred} \beta\left(\mathrm{x}_{2}\right)\right]_{\mathrm{Ev}}\right)_{\mathrm{Fo}}\right]_{\text {Process }}$ 
This is the predication of advenir and arriver. It designates a Process whose only argument of the predication fulfils the semantic role of Force. The complement clause encodes an Event, represented by the variable $e_{1}$. This extensional value is in turn modified by the intension Cert (standing for Certain), which signals that the state of affairs described by the complement is assessed as being the case with no alternatives available ${ }^{6}$. These extensional properties are in turn modified by an intensional value. The intensional properties are codified along a Scale of Evaluation.

The combination of an extensional property and an intensional value gives rise to the complement meaning..

Thus the Force has Certain Event meaning.

2. $\left[\left(\operatorname{Prob} X_{1}:\left[\pi_{2} e_{1}:\left[\pi_{1} \operatorname{Pred} \beta\left(\mathrm{x}_{\mathrm{n}}\right)\right]_{\text {State }}\right.\right.\right.$

This is the predication of seem, appear, look as if, paraitrel and sembler.

This schema designates a State and codifies an argument extensionally characterized as a Fact, represented by the operator $X^{1}$. This extensional value is modified by the variable Prob (standing for Probable). This means that the speaker presents the propositional content as expected although some alternatives are offered.

The complement has Probable Fact meaning.

3. $\left[\left(\mathrm{x}_{1}\right.\right.$ : prototyp. +concrete $\varepsilon$ sign, symbol) $\left(\operatorname{Cert} \mathrm{X}_{1}:\left[\pi_{2} \mathrm{e}_{1}:\left[\pi_{1} \operatorname{Pred} \beta\left(\mathrm{x}_{\mathrm{n}}\right) \pi\right]_{\text {State }}\right.\right.$

This frame designates a State and specifies the relation between a concrete argument performing no semantic function, and a second argument extensionally marked as a Fact $\left(X_{l}\right)$ and intensionally characterized as Certain (Cert). The complement has Certain Fact meaning.

It is the predication of mean, express, indicate / indiquer, and denote.

\section{Contrastive cognitive analysis}

The connections of EXISTENCE with other semantic fields can be formalized in a semantic macronet, where the names of domains in their primary location are written in capital letters.

6. Mairal (1994) has studied the value of the complement of higher predicates. He postulates that the complement of certain predicates can be characterized by both extensional and intensional properties. The former are arranged along a scale of knowledge and behaviour. They indicate what the complement designates in a possible world. Six extensional values are to be distinguished: Quoted Speech Act; Fact; Future Fact; Event; Action, and Individual. 


\section{PERCEPTION:}

1.1. Visual perception:

(i) To exist in the perception of others Exister dans la perception des autres [show, look, look like/as if, date] [se présenter, sembler/paraître1/avoir l'air, se matérialiser]

(ii) To begin to exist in the perception Commencer à exister dans la perception of others, becoming visible des autres, devenant visible [appear, dawn, surface, materialize [apparaître, se former, surgir, jaillir erupt $^{2}$, haunt, form1, arise, come up poindre, se lever, paraître ${ }^{2}$, pointer crop up, form ${ }^{2}$ ] percer, germer]

(iii) To stop existing in the perception of others

Cesser d'exister dans la perception autres

[disappear, evaporate, vanish [disparaître, s'évaporer, se volatiliser fade, dissipate] s'évanouir]

(iv) To cause sth to disappear [remove, rub out, scrape, delete efface, erase, cancel, eliminate ${ }^{2}$ expunge, obliterate, destroy ${ }^{2}$ eradicate]

Faire disparaître complètement qqch [effacer, enlever, éliminer, gommer gratter, raturer, rayer, barrer détruire]

\subsection{Auditory perception:} make $^{2}$, erupt $^{1}$, fade faire, s'élever

\section{FEELING:}

(i) To exist/cause to exist [predominate create, engender, ressurrect]

(ii) To cause sth to exist in time [produce $^{2}$, precipitate, occasion induce]

Faire exister qqch dans le temps [produire $^{2}$, susciter, engendrer ${ }^{2}$ déchaîner]

(iii) To begin/cause to begin to exist Commencer à exister [give birth to, erupt1, germinate1 2] [éclater,exploser,se déchaîner,s'éveiller]

(iv) To begin to exist in the perception of others [materialize, dawn, surface, form]

(v) To continue to exist [live, live on survive, endure, remain]

(vi) To continue to exist in time [linger, persist] 
(vii) To cease to exist / To cause to die Faire mourir qqn/un animal [die, perish, kill, murder, butcher, [tuer, assommer] massacre, slaughter, starve1 2]

(viii) To cease to exist in the perception Cesser d'exister dans la perception of others [disappear, vanish, fade des autres [disparaitre, s'évanouir] dissipate, evaporate]

(ix) To cause sth to disappear

[destroy ${ }^{2}$, obliterate, efface, expunge

remove, erase]

\section{ACTION:}

(i) To cause to exist Faire exister qqn/qqch [make ${ }^{2}$, render, produce1, engender [faire, créer, constituer, produire1 establish, found, ressurrect] établir, fonder, instituer, instaurer ressusciter $\left.{ }^{1}\right]$

(ii) To cause to exist in time Faire exister qqch dans le temps [produce ${ }^{2}$, bring about, spark, induce [produire ${ }^{2}$, causer, provoquer prompt, occasion, effect, provoke] susciter, motiver, occasionner déchaîner, engendrer ${ }^{2}$, déclencher entraîner]

(iii) To cause to begin to exist

Commencer qqch [start ${ }^{2}$, institute, instigate] [commencer ${ }^{2}$, amorcer, engager entamer, entreprendre]

(iv) To cause to begin to exist in time $\left[\right.$ start $^{4}$, initiate, inaugurate, Commencer qqch (Temps) [commencer ${ }^{4}$, inaugurer ${ }^{2}$ ] commence $^{2}$, open $^{2}$, originate $\left.{ }^{2}\right]$

(v) To cause to die Faire mourir qqn/un animal [kill, murder, assassinate, eliminate ${ }^{1}$ bump off, do away with, liquidate exterminate, massacre, butcher [tuer, abattre, assommer, égorger pourfendre, avoir/faire la peau de descendre, assassiner, zigouiller slaughter, execute, behead, decapitate liquider, exterminer, massacrer guillotine, hang, lynch, crucify exécuter, fusiller, décapiter elecrocute, starve ${ }^{2}$, strangle, suffocate ${ }^{2}$ guillotiner, pendre, lyncher smother, gas, drown ${ }^{2}$, commit suicide électrocuter, étrangler, étouffer slay, destroy $\left.{ }^{1}\right]$ noyer, empoisonner, lapider poignarder, crucifier, se suicider se pendre, se brûler la cervelle] 
(vi) To cause sth to disappear [remove, rub out, scrape, delete efface, erase, eliminate, destroy 2 expunge, obliterate, eradicate]
Faire disparaître complètement qqch [effacer, enlever, éliminer, gommer gratter, raturer, rayer, barrer, détruire]

\section{COGNITION:}

(i) To cause to exist

[make ${ }^{2}$, produce1, institute, initiate]

(ii) To cause to begin to exist [loose, miscarry]

(iii) To exist / To begin to exist in the Exister dans la perception des perception of others [seem, appear] [sembler, paraître ${ }^{1}$, avoir l'air]

(iv) To cause sth to disappear [erase, obliterate]

\section{CHANGE:}

(i) To cause to exist [make ${ }^{1}$, render, create]

Faire exister qqn/qqch

(ii) To cause to exist in time [se multiplier,se propager,propager] Faire exister qqch dans le temps [produce ${ }^{2}$, bring about, spark, induce [produire ${ }^{2}$,causer,provoquer, susciter prompt, occasion, effect, provoke] motiver, occasionner, déchaîner engendrer ${ }^{2}$, déclencher, entraîner]

(iii) To begin to exist in the perception of others

Commencer à exister dans la perception des autres [appear,dawn, surface, materialize [apparaître, se former, surgir, jaillir erupt $^{2}$, haunt, form1, arise, come up poindre, se lever, paraître ${ }^{2}$, pointer crop up, form ${ }^{2}$ ] percer, germer] Continuer à exister [se développer grandir, croître, pousser]

\section{POSITION:}

(i) To continue to exist [endure, remain] Continuer à exister [rester ${ }^{2}$ demeurer $^{2}$, se maintenir]

(ii) To continue to exist in time [go on, pass, elapse, last, linger persist, rage]
Continuer à exister dans le temps [continuer, durer, persister, subsister se perpétuer, se prolonger, traîner] 


\section{MOVEMENT:}

To begin/to continue/to cease to exist Commencer/Continuer/Cesser d'exister

\section{EXISTENCE and PERCEPTION}

The verbs under the dimensions To exist in the perception of others, To begin to exist in the perception of others / Commencer à exister dans la perception des autres, To stop existing in the perception of others / Cesser d'exister dans la perception des autres, and To cause sth to disappear / Faire disparaître complètement qqch show a deviation to VISUAL PERCEPTION.

This relation is supported by the following facts:

a) The complementation patterns of several predicates are instances of perceiving something physically:

SV Adjunct (Place)

(1) The edge of the moon is just showing in the sky.

(2) Le soleil parut derrière les collines.

b) The complement meaning of some verbs is semantically marked as "something visible". It is the case of show, appear / apparaître, surface, materialize, form1, se former, surgir, disappear / disparaître, vanish, and dissipate.

c) Some French and English verbs show an extension from physical entities to abstract entities, a deviation which is typical of PERCEPTION verbs.

- Deviation to FEELING: materialize, dawn ${ }^{1}$, surface, germer, disappear / disparaître, dissipate, evaporate, s'évanouir, vanish, and erase.

- Deviation to COGNITION: appear.

\section{EXISTENCE and FEELING}

The connection of EXISTENCE with FEELING is syntax-dependent in that it obtains via the selection restrictions impinging upon the subject/object argument, which is semantically characterized as "feeling":

(3) Sa colère se déchaîna quand il apprit qu'on ne lui avait pas donné le poste qu'il croyait mériter.

In English, the relation of EXISTENCE with FEELING can obtain indirectly. For instance, some verbs in the subdimension To cause to die are indirectly linked to FEELING in that the emphasis is not on the physical action and the result (the death of someone), but on the emotional reaction of the object. In French only two verbs (tuer, assommer) denote this idea:

(4) Elle assomme tout le monde avec ses plaintes et ses reproches.

(5) If I should find out that you have done something behind my back, I'll kill you. 


\section{EXISTENCE and ACTION}

Our claim about the relation of EXISTENCE with ACTION is backed up by the following facts:

a) The verbs make 2 and faire, which belong to the dimensions To cause to exist and Faire exister qqn/qqch are the archilexemes of the semantic domain of ACTION.

b) The predicates within all the causative dimensions (except for the dimensions To cause to die / Faire mourir qqn/un animal and To cause to disappear / Faire disparaître complètement qqch) encode an object semantically marked as "effected" (resulting from the action denoted by the verb). This semantic feature is prototypical of verbs of ACTION.

c) The complement meaning of a few verbs signal a step from physical entities to mental entities. This extension coincides with that postulated for the verbs of ACTION:

- Deviation to FEELING: produce ${ }^{2}$, engender, engendrer ${ }^{2}$, precipitate, occasion, induce, susciter, déchaîner.

- Deviation to AUDITORY PERCEPTION: make², faire.

- Deviation to COGNITION: make ${ }^{2}$, produce ${ }^{1}$, originate1, institute, erase, obliterate, miscarry, loose.

\section{EXISTENCE and COGNITION}

As advanced above, some verbs falling in the dimensions To cause to exist, To cause to begin to exist, and To cause to disappear maintain a close connection with the semantic field of COGNITION in that the semantic scope of their object argument is "intellect":

- Ideas / thoughts / projects: miscarry, produce ${ }^{1}$, erase, obliterate.

- Theories / Institutions: institute, loose.

- Laws: make².

This relation is reinforced syntactically. Some patterns of appear, seem, paraître1 and sembler touch upon MENTAL PERCEPTION.

The form of these government patterns goes as follows:

$\mathrm{SV}$ [PO] O-Nominal Clause
(a) $\mathrm{S}=$ prototyp. it $/ \mathrm{il}$
(b) $\mathrm{O}=$ prototyp. Probable Fact
(6) Il semble qu .
(7) It seems/appears that you are mistaken. 


\section{EXISTENCE and CHANGE}

The complement meaning of some verbs under the dimensions To cause to exist / Faire exister and To cause to exist in time / Faire exister qqch dans le temps signals the transition from one state of affairs to another:

(8) Le coup d'état a entraîné la chute du gouvernement.

(9) All this would precipitate an economic crisis.

(10) The staff reduction has created a lot of problems.

Further, in English the connection of EXISTENCE and CHANGE is reinforced on the syntagmatic level, as shown in the syntactic pattern of the predicate render: SVO Complement / Participle. This complement or participle denotes the state resulting from the action designated by the subject:

(11) The medicine must have rendered her unconscious for a long time.

\section{EXISTENCE and POSITION}

The link of EXISTENCE and POSITION is established via two dimensionallevel schemata: To continue to exist / Continuer à exister, and To continue to exist in time / Continuer à exister dans le temps. The English lexemes in the first dimension focus on the permanence of existence, while the French verbs (rester ${ }^{2}$, demeurer and se maintenir) highlight the continuation of a state of affairs (state or situation).

On the other hand, the verbs within the dimension To continue to exist in time / Continuer à exister dans le temps lay the emphasis on permanence in time.

\section{EXISTENCE and MOVEMENT}

The connection of EXISTENCE with MOVEMENT obtains from the general configuration of the semantic domain of EXISTENCE in both French and English. The field of EXISTENCE is divided into three major dimensions:

- To begin to exist / Commencer à exister

- To continue to exist / Continuer à exister

- To cease to exist / Cesser d'exister

The relation with MOVEMENT results from the conception of existence as a movement through time. 


\section{Codification of metaphorical and metonymical processes}

\begin{tabular}{|c|c|c|c|}
\hline Process & Type & French predicates & English predicates \\
\hline \multirow{3}{*}{ METAPHOR } & Theories are people & & $\begin{array}{l}\text { be, predominate, prevail } \\
\text { be born' }{ }^{1} \text {, arise, originate } \\
\text { institute, initiate, miscarry } \\
\text { flourish, loose, survive } \\
\text { endure, remain, last, linger } \\
\text { persist, go on, die } \\
\text { pass away/on, perish }\end{array}$ \\
\hline & Institutions are people & & $\begin{array}{l}\text { be, predominate, prevail } \\
\text { embody, threaten,originate } \\
\text { be born }^{1} \text {, reincarnate } \\
\text { institute, loose, haunt, arise } \\
\text { initiate, survive, flourish } \\
\text { endure, remain, go on } \\
\text { perish, do away with }\end{array}$ \\
\hline & Machines are people & commencer ${ }^{2}$ & start ${ }^{2}$, die, give up the ghost \\
\hline \multirow[t]{2}{*}{ METONYMY } & Part for the whole & & $\begin{array}{l}\text { originate }^{12} \text {, depict, embody } \\
\text { fake, live, live on, survive } \\
\text { endure, kill }\end{array}$ \\
\hline & Product for producer & & appear, disappear, date \\
\hline \multirow[t]{4}{*}{$\begin{array}{l}\text { DEVIATION FROM } \\
\text { [+CONCRETE] TO } \\
\text { [-CONCRETE] }\end{array}$} & Feeling & $\begin{array}{l}\text { percer, tuer, disparaître, } \\
\text { s'évanouir }\end{array}$ & $\begin{array}{l}\text { predominate, depict, fake } \\
\text { dawn, erupt }{ }^{1} \text {, germinate } \\
\text { give birth to, surface }^{1} \\
\text { materialize, survive, endure } \\
\text { remain, linger, persist, die } \\
\text { pass away/on, perish, kill } \\
\text { disappear, evaporate } \\
\text { vanish, fade, erase, destroy }{ }^{2} \\
\text { eradicate, obliterate } \\
\text { remove, expunge }\end{array}$ \\
\hline & Intellect & & $\begin{array}{l}\text { make }^{2}, \text { produce }^{1} \text {, institute } \\
\text { loose, miscarry, appear } \\
\text { initiate, erase, obliterate }\end{array}$ \\
\hline & $\begin{array}{l}\text { Fact }<\text { situation, event } \\
\text { process, action }>\end{array}$ & & $\begin{array}{l}\text { make }{ }^{12} \text {, render, create } \\
\text { depict, reproduce } \\
\text { materialize, produce } \\
\text { occasion, bring about } \\
\text { induce, provoke, prompt } \\
\text { go on, last, suffocate, kill } \\
\text { do away with }\end{array}$ \\
\hline & Sound & faire & make $^{2}$, erupt ${ }^{1}$ \\
\hline
\end{tabular}

We observe that metaphorical and metonymical processes are relevant to the cognitive structure of the domain of EXISTENCE in English, while in French there is a poor codification of these processes. 


\section{Conclusions}

In conclusion, the lexicon is not an inconsistent inventory of words, but a structured whole of semantically bound units which are grouped under semantic domains structured paradigmatically, syntagmatically, pragmatically and cognitively.

The contrastive analysis of the French and English domains of EXISTENCE has highlighted the relations of equivalence between the French and English lexemes. We can conclude that the similarities between the French and English fields of EXISTENCE are far more prominent than the divergences on the different levels of analysis:

1) On the paradigmatic level, we have noted that the same relevant differentiation parameters traverse the French and English domains.

2) On the syntagmatic level, the examination of the predicate schemata encoded in the French and English fields has revealed that the verbs pattern syntactically in the same way.

3) On the cognitive level, the description of the semantic links of both domains has yielded the same metaphorical projections from these fileds into others.

In sum, starting from the idea that semantic structure reflects conceptual structure, we claim that the way of interpreting reality is roughly the same across culture boundaries.

\section{References}

Coseriu, E. (1977). Principios de semántica estructural. Madrid: Gredos.

DIK, S.C. (1978a). Functional grammar. Dordrecht: Foris Publications.

DIK, S.C. (1978b). Stepwise Lexical Decomposition. Lisse: The Peter de Ridder Press.

FABER, P. (1994a). "The semantic architecture of the lexicon: a study in the configuration of lexical fields". Lexicographica, Series Maior 57. Tübingen: Max Niemeyer, 37-51.

FABER, P. and MAIRAL, R. (1994). "Methodological underpinnings for the construction of a Functional-Lexicological model”. Miscelánea, A Journal of English and American Studies. Vol. 15, 193-217.

FABER, P. \& MAIRAL, R. (1997a). "The paradigmatic and the syntagmatic structure of the semantic field of EXISTENCE in the elaboration of a semantic macronet". In Studies in Language, 21:1, 119-154. Amsterdam: John Benjamins.

FABER, P. and MAIRAL, R. (1998). “Towards a typology of predicate schemata". In Wotjak, G. (ed.), Functional Lexicology. Peter Lang.

LAKoff, G. (1987). Women, fire and Dangerous Things. What Categories Reveal about the Mind. Chicago: University of Chicago Press. 
LAKoff, G. \& Johnson, M. (1980). Metaphors we live by. Chicago: University of Chicago Press.

Lyons, J. (1977). Semantics. Volumes I and II. Cambridge University Press.

MAIRAL, R. (1994). "Parámetros para la organización de una sintaxis léxica funcional”. In J. Martín Arista (ed.), Estudios de Gramática Funcional. Zaragoza: Mira, 23-73.

Martin Mingorance, L. (1984). "Lexical fields and Stepwise Lexical decomposition in a Contrastive English-Spanish verb Valency Dictionary”. In R.R.K. Hartman (ed.), LEXeter'83 Proceedings. $\quad$ Papers from the International Conference on Lexicography at Exeter. Tübingen: Max Niemeyer., 226-236.

Martin Mingorance, L. (1990). "Functional grammar and lexematics in lexicography”. In J. Tomaszczyk and B. Lewandoska-Tomaszczyk (eds.), Meaning and Lexicography. Amsterdam: John Benjamins., 227-253.

Martin Mingorance, L. (1990). "Léxico y sintaxis en la gramática funcional de S.C.Dik”. Cuadernos de Investigación Filológica. Logroño: CUL.

Sommers, H.L. (1984). "On the validity of the complement-adjunct distinction in valency grammar". Linguistics 22, 507-530.

\section{Appendix 1: Semantic classification of EXISTENCE verbs in French and English}

1. The parameter of Manner

\begin{tabular}{|c|c|}
\hline \multicolumn{2}{|r|}{ English } \\
\hline Former & \\
\hline \multicolumn{2}{|l|}{ Etablir } \\
\hline Fonder & Found \\
\hline \multicolumn{2}{|l|}{ Constituer } \\
\hline \multicolumn{2}{|l|}{ Se reproduire ${ }^{1}$} \\
\hline & Look \\
\hline \multicolumn{2}{|l|}{ Paraître ${ }^{1}$} \\
\hline & Seem \\
\hline \multicolumn{2}{|l|}{ Sembler } \\
\hline \multicolumn{2}{|l|}{ Avoir l'air } \\
\hline \multicolumn{2}{|l|}{ Se matérialiser } \\
\hline & Personify \\
\hline & Embody \\
\hline Copier & Copy \\
\hline \multicolumn{2}{|l|}{ Survenir } \\
\hline & Happen \\
\hline Advenir & Occur \\
\hline \multicolumn{2}{|l|}{ Eclater } \\
\hline Exploser & Erupt $^{1}$ \\
\hline Se déchaîner & \\
\hline
\end{tabular}


Eclore

Se former

Pointer

Percer

Germer

S'élever

Se développer

Prospérer

S'épanouir

Abattre

Assommer

Etrangler

Etouffer

Egorger

Massacrer Massacre

Empoisonner

Lapider

Fusiller

Décapiter

Pendre

Crucifier

Se pendre

Raturer

Barrer

Se volatiliser
Hatch

Make $^{2}$

Materialize

Erupt $^{2}$

\section{Crop up}

Rage

Do away with

Liquidate

Strangle

Suffocate ${ }^{2}$

Smother

Gas

Behead

Hang

Crucify

Electrocute

Starve 12

Drown ${ }^{1} 2$

Remove

Rub out

Scrape

Delete

Efface

Erase

Cancel

Vanish 
Se dissiper

Fade

S'évaporer

S'évanouir

Dissipate

2. The parameter of Means / Instrument

Exprimer (Means)

Express (Means)

Symboliser (Means)

Symbolize (Means)

Dénoter (Means)

Denote (Means)

Personnifier (Means)

Exemplify (Means)

Incarner (Means)

Incarnate (Means)

Egorger (Instr.)

Poignarder (Instr.)

Guillotiner (Instr.)

Gratter (Instr.)

Guillotine (Instr.)

Gommer (Instr.)

3. The parameter of Time

Coexister

S'ensuivre Ensue (from)

Resurrect

Look like/as if

Avoir lieu Take place

Coïncider

Coincide

Se reproduire ${ }^{2}$

Resume

Threaten

Ressusciter ${ }^{2}$

Reincarnate

Avorter /

Faire une fausse couche

Miscarry

Live on

Survivre

Survive

Hanter

Surgir

Come up

Jaillir

Durer

S'écouler/Passer

Pass

Persister

Subsister

Se perpétuer

Se prolonger

Linger

Traîner

Persist

4. The parameter describing the nature of the subject / object

4.1. Identity of the subject

Procréer

Eclore

Procreate

Hatch 
S'éveiller

Poindre

Se lever

Paraître ${ }^{2}$

Pointer

Dawn

Percer

Germer

S'élever

Hanter

Fleurir

Prospérer

Grandir

Croître

Pousser

Erupt $^{2}$

4.2. Identity of the object

Inaugurer

Instituer

Etablir

Establish

Instaurer

Pondre

Engender

Se reproduire ${ }^{1}$

Lay

Exprimer

Reproduce

Personnifier

Personify ${ }^{2}$

Incarnate

Falsifier

Contrefaire

Imiter

Duplicate

Counterfeit

Forge

Déchaîner

Avorter /

Faire une fausse couche

Amorcer

Engager

Entreprendre

Entamer

Assassinate

Eradicate 


\subsection{Quality of the object}

Créer

Create

Produire ${ }^{1}$

Befall

Occur

Occasionner

Provoke

Loose

Form ${ }^{1}$

Crop out

Végéter

Vivoter

Rub out

Erase

5. The parameter of Place

Prevail

Grow

Coexister

Avoir lieu

Depict

Coïncider

Take place

Coincide

Se réincarner

Reincarnate

Se lever

Hanter

Haunt

S'épanouir

Gratter

Rayer

\section{Appendix 2: Paradigmatic structure of the domain of EXISTENCE in French}

\section{EXISTER}

être: exister (philosophique).

coexister: exister dans le même lieu au même moment.

\section{FAIRE EXISTER}

faire: faire exister qqch.

créer: faire exister qqn/qqch de nouveau.

constituer: créer qqch conformément à la loi.

produire1: faire exister qqch qui n'existe pas encore.

se reproduire': produire des êtres semblables à soi par la génération.

se multiplier/se propager/propager: se reproduire, en faisant augmenter le nombre. procréer: produire des êtres vivants par voie de génération (littér.).

engendrer ${ }^{1}$ : procréer (le mâle). 
pondre: faire des oeufs (les animaux ovipares, spéc. les poules).

former: faire exister qqch en lui donnant une forme/organisation particulière.

établir: faire exister qqch (une institution/loi/règle) en le mettant en application.

fonder: établir qqch en fournissant parfois une somme.

inaugurer: établir qqch (une pratique/un usage).

instituer: établir qqch (un tribunal/un usage/une règle).

instaurer: établir qqch (une institution/un régime politique/une loi/une règle/une mode).

ressusciter ${ }^{1}$ : faire exister de nouveau qqch qui avait disparu.

EXISTER DANS LA PERCEPTION DES AUTRES

se présenter: exister dans la perception des autres.

paraître1/sembler/avoir l'air: se présenter sous un certain aspect.

se matérialiser: se présenter sous une apparence matérielle/visible.

\section{EXISTER EN TANT QUE REPRESENTATION DE QQCH}

représenter: exister en tant que représentation de qqch.

exprimer: représenter une idée/un sentiment au moyen du langage ou de l'art.

symboliser: représenter qqch par un symbole.

dénoter: représenter qqch en tant que signe.

indiquer: être le signe d'une autre chose.

personnifier: représenter une abstraction.

incarner: personnifier qqch sous une forme matérielle et visible.

\section{FAIRE EXISTER QQCH EN TANT QUE REPRESENTATION D'UNE AUTRE CHOSE}

reproduire: faire une représentation de qqch.

copier: reproduire fidèlement qqch.

falsifier: reproduire qqch (monnaie/une chose écrite) dans une intention frauduleuse.

contrefaire: reproduire qqch (un objet/écriture/monnaie) dans une intention frauduleuse.

imiter: reproduire qqch (monnaie/écriture/une chose écrite) dans une intention frauduleuse.

\section{EXISTER DANS LE TEMPS}

se produire/arriver/se passer: commencer à exister dans le temps.

survenir: se produire de façon inattendue et brusque.

advenir: se produire de façon imprévisible.

s'ensuivre: se produire à la suite de qqch.

avoir lieu: se produire à un endroit ou à un moment donné.

coïncider: se produire dans le même lieu au même moment.

se reproduire ${ }^{2}$ : se produire une nouvelle fois.

devenir: arriver qqch à qqn (emploi à la forme interrogative).

FAIRE EXISTER QQCH DANS LE TEMPS

produire ${ }^{2} /$ causer/provoquer/susciter/motiver/engendrer $^{2}$ : faire exister qqch dans le temps

occasionner: produire qqch de fâcheux.

déchaîner: provoquer une certaine réaction.

entraîner: provoquer en ayant pour effet qqch. 
COMMENCER A EXISTER/VIVRE

commencer²: commencer à exister.

éclater: commencer de façon soudaine et claire.

exploser: commencer brusquement et violemment.

se déchaîner: commencer avec une grande violence.

naître1: commencer à exister/vivre.

naître ${ }^{2}$ : naître avec certaines caractéristiques.

éclore: commencer à exister en sortant de l'oeuf (les animaux ovipares).

s'éveiller: commencer à exister (une idée/un sentiment).

ressusciter ${ }^{2}$ : naître de nouveau après être mort.

se réincarner: naître dans un autre corps après être mort.

\section{FAIRE EXISTER QQCH/DONNER NAISSANCE A QQN}

commencer $^{2}$ : faire exister qqch.

amorcer: commencer qqch (une conversation/une affaire).

engager: commencer qqch (une bataille/une affaire/une conversation/des négociations).

entamer: commencer qqch (un sujet/une affaire/une conversation/des relations).

entreprendre: commencer qqch demandant de longs efforts/une coordination.

accoucher/donner le jour: donner naissance à qqn.

enfanter: donner naissance à qqn (litt.).

avorter/faire une fausse couche: accoucher avant terme d'un foetus/enfant mort.

COMMENCER A EXISTER DANS LA PERCEPTION DES AUTRES

apparaître: commencer à exister dans la perception des autres.

hanter: apparaître souvent quelque part (les esprits/fantômes).

se former: apparaître en prenant une certaine forme.

surgir: apparaître soudainement.

s'élever: surgir en augmentant d'intensité (un bruit/une voix).

jaillir: apparaître soudainement/abondamment.

poindre: apparaître (la lumière du jour ou d'un astre).

se lever: apparaître à l'horizon (un astre).

paraître ${ }^{2}$ : apparaître à l'horizon (soutenu).

pointer: apparaître peu à peu (la lumière/les étoiles).

percer: apparaître au moyen de plusieurs signes (le soleil/un sentiment).

germer: apparaître en se développant (un projet).

\section{CONTINUER A EXISTER}

vivre: continuer à exister (les êtres animés).

végéter: vivre une vie difficile/insipide/médiocre.

vivoter: vivre une vie précaire/morne.

survivre: vivre encore après un évènement passé/désagréable.

se développer: continuer à exister en devenant plus grand/meilleur.

fleurir: se développer (une plante/une activité humaine).

prospérer: se développer rapidement (une activité économique).

s'épanouir: se développer harmonieusement dans le domaine moral/affectif/intellectuel.

grandir: continuer à exister en devenant plus grand (les êtres humains). 
croître: continuer à exister en devenant plus grand (animaux/plantes). pousser: continuer à exister en devenant plus grand (la végétation). rester1: continuer à exister (après élimination des autres éléments). rester': continuer à être dans tel état/situation/disposition. demeurer: continuer à être dans tel état/situation/disposition (soutenu). se maintenir: continuer à être dans le même état.

\section{CONTINUER A EXISTER DANS LE TEMPS}

continuer: continuer à exister dans le temps.

passer/s'écouler: continuer, en arrivant à son terme.

durer: continuer pendant un certain temps.

persister: durer (insistance sur l'idée de continuité)

subsister: durer après élimination des autres éléments ou malgré l'écoulement du temps.

se perpétuer: durer longtemps.

se prolonger: durer plus longtemps qu'il n'était prévu/prévisible.

traîner: durer trop longtemps.

\section{CESSER D'EXISTER}

mourir: cesser d'exister ou de vivre.

décéder: mourir (style juridique/administratif).

claquer: mourir (familier, populaire).

crever: mourir (fam., pop.).

casser sa pipe: mourir (fam., pop.).

passer l'arme à gauche: mourir (fam., pop.).

manger les pissenlits par la racine: mourir (fam., pop.).

avaler son bulletin de naissance/sa chique: mourir (fam., pop.).

sortir les pieds devant/sortir entre quatre planches: mourir (fam., pop.).

boire le bouillon d'onze heures: mourir (fam., pop.).

déposer le bilan: mourir (fam., pop.).

faire couic: mourir (fam., pop.).

ramasser ses outils: mourir (fam., pop.).

faire sa malle/valise: mourir (fam., pop.).

aller chez les taupes: mourir (fam.,pop.).

expirer1: mourir (soutenu).

périr: mourir (littéraire)

rendre l'âme: mourir (vieilli).

se noyer: mourir accidentellement par immersion.

se tuer: mourir dans un accident.

s'électrocuter: mourir accidentellement par une décharge électrique.

\section{FAIRE MOURIR QQN/UN ANIMAL}

tuer: faire mourir qqn/un animal de mort violente.

abattre: tuer qqn/un animal en le faisant tomber à terre.

assommer: tuer qqn/un animal en le frappant violemment sur la tête.

égorger: tuer qqn avec une arme tranchante/tuer un animal en lui coupant la gorge.

avoir/faire la peau de: tuer qqn (pop.). 
assassiner: tuer qqn avec préméditation/par guet-apens.

zigouiller: tuer/assassiner qqn (pop.).

massacrer: tuer avec sauvagerie et en grand nombre des personnes qui ne peuvent se défendre.

exterminer: tuer entièrement jusqu'au dernier.

liquider: tuer qqn pour s'en débarrasser.

exécuter: tuer qqn en vertu d'un jugement.

fusiller: exécuter qqn par une décharge de coups de fusil.

décapiter: exécuter qqn en lui tranchant la tête.

guillotiner: décapiter qqn au moyen d'un instrument de supplice servant à trancher sa tête.

pendre: exécuter qqn en le suspendant par le cou au moyen d'une corde.

crucifier: exécuter qqn en le suspendant à une croix.

électrocuter: exécuter qqn par une décharge électrique.

lyncher: tuer qqn sans jugement régulier.

étrangler: tuer qqn en lui opprimant la gorge pour l'empêcher de respirer.

étouffer: tuer qqn par asphyxie.

noyer: étouffer qqn en le plongeant dans un liquide.

empoisonner: tuer qqn en lui faisant absorber du poison.

lapider: tuer qqn à coups de pierre.

poignarder: tuer qqn avec un poignard/un couteau.

se suicider: se tuer par une action volontaire.

se brûler la cervelle: se suicider (fam.).

se pendre: se suicider en suspendant une corde à son cou.

CESSER D'EXISTER DANS LA PERCEPTION DES AUTRES

disparaître: cesser d'exister dans la perception des autres.

s'évaporer: disparaître subitement (qqn) à l'insu des autres (fam.).

se dissiper : disparaître progressivement.

se volatiliser: disparaître très rapidement.

s'évanouir: disparaître sans laisser de traces.

FAIRE DISPARAITRE COMPLETEMENT QQCH

effacer/enlever/éliminer: faire disparaître complètement qqch.

gratter: effacer qqch sur une surface frottée avec qqch de dur.

gommer: effacer qqch avec une gomme.

raturer: effacer qqch par des ratures.

rayer: effacer le nom de qqn d'un ensemble/d'une liste/d'un registre.

barrer: rayer qqch d'un trait pour l'annuler.

détruire: effacer qqch de sorte qu'il n'y ait plus de traces.

\section{CESSER D'EXISTER DANS LE TEMPS}

finir/(se) terminer/cesser/s'achever: cesser d'exister dans le temps.

expirer $^{1}$ : finir parce que cela est arivé à son terme. 


\section{Appendix 3: Paradigmatic structure of the English domain of EXISTENCE}

TO EXIST

be: to exist (formal) (technical).

prevail: to exist everywhere as common/general.

grow: to exist esp. in a particular place (plants).

predominate: to exist in greater numbers/degree/importance.

\section{TO CAUSE TO/MAKE EXIST}

make': to cause to be.

render: to make (formal).

create: to cause to/make exist (sth new/original).

make2: to create, forming it by a work/action.

beget 1 : to create sth (formal) (old-fashioned).

produce1: to create (emphasis on the result/effect).

engender: to produce sth (usu. a situation/condition/atmosphere/feeling) (formal).

reproduce 1 : to produce offspring (animals/people/plants).

procreate: to reproduce (animals/people) (formal).

beget $^{2}$ : to procreate (esp. of a male parent) (formal) (old-fashioned).

sire: to beget as the father (old-fashioned).

propagate: to reproduce (animals), thus increasing in numbers (formal).

lay: to produce eggs (of insects and esp. hens).

spawn: to lay eggs in large quantities together (of fishes and frogs).

establish: to create sth (system/organization/state of affairs).

found: to establish usu. by supplying money.

resurrect: to cause sth to exist again after having disappeared.

\section{TO EXIST IN THE PERCEPTION OF OTHERS}

show: to exist in the perception of others.

seem: to show as.

look: to seem by expression/appearance.

look like/as if: to seem to be going to.

date: to seem to be (old-fashioned).

\section{TO EXIST AS A REPRESENTATION OF SOMETHING}

represent: to be a sign/picture/symbol/example of sth.

stand for: to represent (informal).

mean: to represent sth/a likely result of sth.

express: to represent by a symbol/character/formula, etc.

signify: to represent sth, being a sign of it (formal).

indicate: to be a sign of sth else.

symbolize: to represent sth by being a symbol of it.

denote: to represent sth by being a sign/symbol of it.

exemplify: to represent by being an example of.

instance: to exemplify (formal).

personify2: to exemplify sb/sth perfectly.

typify: to represent as a genuine symbol/characteristic of. 
depict: to represent in a painting/drawing. embody: to represent by including/comprising.

personify2: to represent sth abstract/inanimate in the form of a person in literature (tech). incarnate: to represent a concept/quality in human form.

TO CAUSE TO EXIST AS A REPRESENTATION OF STH

copy: to make sth exactly like another thing.

duplicate: to make an exact copy of sth, usu. sth written/drawn.

reproduce 2 : to make a fairly accurate copy.

fake: to reproduce sth to make it appear genuine, esp. to trick/deceive others.

forge: to reproduce sth exactly, usu. documents, handwriting, paintings in order to deceive.

counterfeit: to reproduce sth, usu. money in order to deceive.

\section{TO EXIST IN TIME}

happen: to be/become real in time (of events).

take place: to happen in a particular place or at a given time.

transpire: to happen.

occur: to happen (esp. of unplanned events) (formal).

befall: to happen (esp. of unpleasant events) (formal) (literary).

coincide (with): to happen at the same time.

resume: to happen again after a pause.

ensue (from): to happen after/as a result (formal).

become of: to happen to (esp. as time passes), in (direct/indirect) questions.

threaten: to seem to be about to happen.

\section{TO CAUSE STH TO EXIST IN TIME}

produce $^{2}$ : to cause sth to happen.

bring about: to cause sth to happen.

occasion: to bring about (formal).

effect: to bring about (formal).

spark: to cause sth to happen, being the direct cause of it.

prompt: to cause sth to happen.

provoke: to cause sth often violent/unpleasant to happen.

\section{TO BEGIN TO EXIST}

start $^{1}$ : to begin to exist/do/feel.

originate 1 : to begin to exist.

commence 1 : to begin to exist (formal).

$\operatorname{erupt}^{1}$ : to begin suddenly.

develop1: to begin to exist, becoming larger/fuller/better.

germinate $^{1}$ : to develop (formal) (literary).

dawn1: to begin to develop (formal).

be born 1 : to begin to exist by birth (living beings).

be born': to be born of particular circumstances.

be born 3 : to be born with certain characteristics . 
hatch: to be born by coming/breaking out of an egg.

reincarnate: to be born again in the body of $\mathrm{sb} / \mathrm{sth}$ else after having died.

\section{TO CAUSE TO BEGIN TO EXIST}

start $^{2}$ : to cause sth to begin to exist.

originate $^{2}$ : to cause sth to begin to exist.

institute: to start sth, bringing it into being (formal).

instigate: to start sth, causing it to be by your own efforts.

commence $^{2}$ : to cause sth to begin (formal).

loose: to cause sth (usu. sth unpleasant) to begin (literary).

develop $^{2}$ : to cause sth to begin to exist, becoming larger/fuller/better.

germinate $^{2}$ : to develop sth (formal) (literary).

give birth to: to cause $\mathrm{sb} / \mathrm{sth}$ to / make sb/sth begin to exist.

bear: to give birth to, producing young/fruit.

produce $^{3}$ : to bear (as the result of a natural process).

deliver: to help a woman give birth.

miscarry: to give birth too early for life to be possible.

\section{TO BEGIN TO EXIST IN THE PERCEPTION OF OTHERS}

appear: to begin to exist in the perception of others.

dawn': to begin to appear (formal).

surface: to appear, becoming obvious/known.

materialize: to appear suddenly, after being invisible.

erupt $^{1}$ : to appear suddenly (of a rash/sore).

haunt: to appear/be regularly in a certain place, usu. causing fear/problems/worry.

form 1 : to appear, beginning to be visible and having a clear shape/outline.

arise: to begin to exist in the perception of others.

come up: to arise unexpectedly.

crop up: to arise suddenly/unexpectedly (usu. sth difficult/unpleasant) (informal).

form $^{2}$ : to begin to exist in the perception of others and develop.

\section{TO BEGIN TO EXIST IN TIME}

start $^{3}$ : to begin to happen.

commence ${ }^{3}$ : to begin to happen (formal).

open1: to start (of a public event).

originate $^{3}$ : to begin to happen (formal).

TO CAUSE TO BEGIN TO EXIST IN TIME

start $^{4}$ : to cause sth to begin to happen.

initiate: to start sth.

open ${ }^{2}$ : to start (of a public event).

inaugurate: to start sth, establishing it firmly (esp. with an official ceremony).

induce: to cause sth to begin to happen.

commence 4 : to cause sth to begin to happen (formal).

\section{TO CONTINUE TO EXIST}

live: to continue to exist (living beings). 
live on: to continue to live, usu. for a long time (emphatic).

survive: to continue to live longer than/after (esp. after coming close to death).

flourish: to continue to exist being successful/strong/healthy.

endure: to continue to exist without loss in quality/importance.

remain: to continue to exist esp. when similar things do not.

\section{TO CONTINUE TO EXIST IN TIME}

go on: to continue to exist in time (events) esp. over a length of time. pass: to go on/by, beginning, continuing and finishing.

elapse: to pass (formal).

last: to continue to exist in time.

linger: to continue to exist for a longer time than people expected.

persist: to continue to exist, esp. after trying to make it disappear.

rage: to continue to exist with great violence (of a storm/fire/battle).

\section{TO CEASE TO EXIST}

die: to cease to exist.

expire$^{1}$ : to die (literary).

pass away/on: to die (euphemism).

kick the bucket: to die (informal).

give up the ghost: to die (old-fashioned).

perish: to die as a result of very hard conditions (formal) (literary).

drown 1 : to die by being under the water and unable to breathe.

be killed: to die as a result of an accident.

suffocate ${ }^{1}$ : to die from lack of air to breathe.

starve1: to die from lack of food.

\section{TO CAUSE TO DIE}

kill: to cause to die.

murder: to kill sb on purpose and unlawfully, using violence.

assassinate: to murder an important politician, for political reasons/reward.

eliminate $^{1}$ : to murder $\mathrm{sb}$ (informal, euphemism).

do in: to kill/murder sb (very informal).

bump off: to kill/murder sb (very informal).

do away with: to kill/murder sb, esp. secretly (informal).

liquidate: to do away with sb (informal, euphemism).

exterminate: to kill all the animals/people (esp. a certain group/race) in a place.

massacre: to kill (esp. defenceless) people in large numbers on a given occasion, in a cruel way.

butcher: to kill animals preparing them for food.

slaughter: to butcher in large numbers.

execute: to kill $\mathrm{sb}$ as a punishment for a serious crime.

behead: to execute sb by cutting off their head.

decapitate: to behead (formal).

guillotine: to execute sb using a guillotine (a device used in France, esp. in the past with a sharp blade tocut off sb's head). 
hang: to execute by tying a rope around their neck and taking away the support under their feet.

lynch: to hang sb without a lawful trial.

crucify: to execute sb by tying/nailing them on a cross and leaving them to die.

electrocute: to execute sb by means of an electrical apparatus.

starve2: to kill sb from lack of food.

strangle: to kill sb by squeezing their throat so that they cannot breathe.

suffocate 2 : to kill sb by stopping their supply of air.

smother: to suffocate sb by covering their face with sth.

gas: to kill sb by making them breathe poisonous gases.

drown': to kill sb by keeping them under the water so that they are unable to breathe.

commit suicide: to kill oneself purposefully.

slay: to kill (poet) (BrE).

destroy': to kill an animal usu. because it is dangerous/ill.

TO CEASE TO EXIST IN THE PERCEPTION OF OTHERS

disappear: to cease to exist in the perception of others.

evaporate: to disappear (feeling).

vanish: to disappear suddenly in a way that cannot be explained.

fade: to disappear gradually, often remaining to some extent.

dissipate: to disappear completely.

TO CAUSE STH TO DISAPPEAR

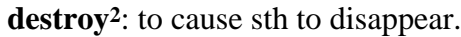

remove: to cause sth to disappear by taking it away/off/out.

rub out: to remove a mark/writing by moving sth on the surface of sth.

scrape: to remove sth from a surface by pushing sth rough along it.

delete: to remove sth by rubbing/striking/cutting it out.

efface: to remove sth by rubbing/wiping (formal).

erase: to remove writing/sound from a surface/ tape by rubbing it out.

eliminate2: to remove sth completely, esp. because unnecessary or unwanted (formal).

expunge: to remove sth completely because it causes problems/bad feelings.

obliterate: to remove all signs of sth.

eradicate: to cause sth bad to disappear (crime, disease).

\section{TO STOP HAPPENING}

end: to stop, continuing no further.

expire $^{2}$ : to come to an end (usu. after a particular length of time).

finish: to end, usu. esp. being completed.

conclude: to finish, coming to an end (formal).

finalize: to finish, becoming final/complete.

terminate: to end officially/definitely/suddenly.

lapse: to stop, coming to an end. 